\title{
LA CIENCIA COMO DISCURSO: \\ ESTUDIOS DEL DISCURSO CIENTÍFICO Y ACADÉMICO \\ Entrevista a Anamaría Harvey Arellano
}

\author{
Oscar Iván Londoño Zapata* \\ Universidad del Tolima
}

Es un honor para mí rendir un homenaje a una de las académicas pioneras en el desarrollo de los Estudios del Discurso (ED) en América Latina: la Dra. Anamaría Harvey Arellano, quien fue Profesora Titular Adjunta de la Pontificia Universidad Católica de Chile. Tuve la oportunidad de realizarle esta entrevista a través de comunicación electrónica escrita durante varios meses del año 2011. En este diálogo, la doctora Harvey volvió la mirada atrás y reflexionó sobre lo que fue una larga y prominente carrera académica. De esta manera, compartió con agrado sus experiencias de formación universitaria, así como sus principales planteamientos acerca de los Estudios del Discurso (ED) y sus aplicaciones en los campos de la divulgación científica y la academia.

\section{Discurso, ciencia y academia}

Si partimos de la idea de que uno de los aspectos que caracteriza y define el mundo académico es la construcción de comunidades científicas cuyos miembros propician la producción y la circulación del conocimiento, debemos reconocer el papel fundamental de la formación escolar y del lenguaje en tal proceso. Es por ello que este universo de saberes se conforma y transforma a través de la ciencia y la alfabetización disciplinar y, de igual manera, por medio de la creación y el uso de estrategias discursivas particulares.

De esta forma, diversas ciencias del lenguaje han virado sus miradas hacia el estudio de los discursos científico y académico, como

\footnotetext{
Licenciado en Lengua Castellana y Magíster en Educación de la Facultad de Ciencias de la Educación de la Universidad del Tolima. Miembro de la Asociación Latinoamericana de Estudios del Discurso, ALED. Docente de Tiempo Completo del Departamento de Estudios Interdisciplinarios del Instituto de Educación a Distancia (IDEAD) de la Universidad del Tolima, Colombia.
} 
manifestaciones del lenguaje en uso fundamentales en los procesos de (re)construcción y apropiación del saber. Es por ello que al tener claro que una de las metas de la mayoría de instituciones de educación superior es facilitar en los estudiantes la generación del conocimiento científico desde miradas disciplinares, transdisciplinares y críticas, entra en juego el desarrollo de procesos de producción y recepción de textos, que orientados hacia tal interés, estructuran el universo discursivo de la escena científica y disciplinar.

El Análisis del Discurso (AD), como campo de investigación del lenguaje en sociedad, ha orientado sus intereses investigativos hacia el estudio de los discursos académico y científico, y es así como desde esta perspectiva, la ciencia y la academia no solo se conciben como prácticas sociales, culturales e históricas sino que son significadas como actividades discursivas que poseen determinados dispositivos de producción, circulación y recepción. Es entonces una tarea del Análisis del Discurso (AD) develar la constitución lingüística, discursiva, social, política e ideológica de la ciencia y la academia.

Anamaría Harvey Arellano se destacó por ser una de las académicas chilenas pioneras en el desarrollo de los Estudios del Discurso (ED) en su país y en América Latina, debido a que aportó significativamente en el estudio discursivo de la ciencia, a través de propuestas teóricas y aplicadas. La doctora Harvey estudió la manera como se conceptualiza y actualiza discursivamente la (co)construcción social del conocimiento en tres ámbitos principales, los cuales indagó en el transcurso de su prominente carrera académica: al interior de la comunidad científica, en la academia y en la prensa escrita.

Algunos de los intereses investigativos de Anamaría giraron en torno al estudio de las representaciones del género informe (Harvey \& Muñoz, 2006) en el discurso académico, es decir, el análisis de las percepciones de los docentes respecto de las características retórico-funcionales del género informe. Asimismo, estudió la entrevista de divulgación científica (Harvey \& Granato, 2000), y la intersubjetividad y el consenso en el diálogo en escenas escolares (Harvey \& Fant, 2008). De igual manera, reflexionó sobre las manifestaciones evaluativas en la ciencia como discurso (Harvey, 2005a) y acerca de la evaluación en el discurso de los informes escritos por estudiantes universitarios (Harvey, 2005b). 
Las constantes preocupaciones de Anamaría por este amplio campo de abordaje del discurso hicieron que orientara sus reflexiones hacia la pertinencia del proceso de alfabetización académica y sus manifestaciones discursivas en las universidades. Para Harvey (2009):

Estudiar la alfabetización académica en toda su complejidad implica, primeramente, aceptar que nos enfrentamos a una práctica social en la cual convergen ideologías disciplinares, esquemas de pensamiento y variedades de lenguaje (Harvey, Núñez y Oyanedel, 2006). También es necesario entender que el proceso de aprendizaje se plasma en las variadas interacciones en que docentes y estudiantes participan, algunas escritas y otras orales, en los distintos estadios de formación de sus respectivas comunidades de práctica. (p. 628)

Es por ello que resulta importante analizar la manera como se puede facilitar la inserción de los estudiantes en la cultura académica, así como las formas en que interactúan los alumnos con esta cultura y sus respectivos géneros discursivos.

Estos y otros temas y problemas motivaron el interés de Anamaría, quien a partir de su trabajo con un equipo de académicos y estudiantes del departamento de Ciencias del Lenguaje de la Pontificia Universidad Católica de Chile, logró construir una original propuesta de abordaje del discurso académico. Una perspectiva orientada al estudio del discurso académico disciplinar oral, escrito y multimodal, mediante una postura investigativa que contempla distintas fases y un recorrido metodológico asociado, que implica recabar información de distintas fuentes en forma paralela.

De esta manera, Anamaría Harvey Arellano deja un invaluable legado académico e investigativo no solo a los analistas del discurso chilenos sino también a las nuevas generaciones de estudiantes y profesionales de las ciencias del lenguaje y las ciencias humanas y sociales latinoamericanos que deciden iniciar sus caminos académicos por el sendero de los Estudios del Discurso (ED). 


\section{En torno al discurso y los Estudios del Discurso (ED)}

Oscar Iván Londoño Zapata (OILZ): ¿Cómo surgió su interés por los estudios del lenguaje y del discurso?

Anamaría Harvey Arellano (AHA): Responder esta pregunta no es fácil. Es como tratar de explicar de qué manera una primera mirada se trasforma en atracción y, posteriormente, en enamoramiento. En mi caso hubo circunstancias, maestros, oportunidades y contactos que hicieron que una motivación inicial pudiera desarrollarse y transformarse en el aspecto nuclear de mi quehacer académico.

Mi interés por el lenguaje surgió cuando opté por estudiar pedagogía en inglés en la Facultad de Filosofía y Educación de la Universidad de Chile, y por el discurso, prácticamente cuando todavía era estudiante en esa casa de estudios y fui nombrada ayudante. Bajo este cargo tuve que orientar clases de lo que en ese entonces se denominaba inglés instrumental y se conociera después como ESP (English for Specific Purposes), a estudiantes de biología y matemáticas y tuve que empezar a familiarizarme con el discurso académico y especializado. Una vez titulada, ya como profesora en las sedes de provincia, primero, y en la Facultad de Ciencias Físicas y Matemáticas de la misma universidad, después, comencé a estudiar y a describir las características del discurso científico y de ciertos géneros discursivos con el propósito de desarrollar la comprensión lectora en lengua extranjera. Materiales y textos de estudio fueron el producto de esos esfuerzos iniciales. Posteriormente, comencé a realizar investigaciones formalizadas más ambiciosas en estas mismas áreas.

Durante esos años participé activamente en una organización latinoamericana de profesores de inglés especializado, cuyo primer encuentro científico se realizó en Paipa (Colombia) en el año 1977, con el patrocinio del Consejo Británico. Este foro, que ha tenido como sedes a Colombia, Brasil, México, Argentina, Venezuela y Chile, y en el cual estuve presente hasta el año 1998, me permitió, además, conocer la realidad académica de distintos países latinoamericanos. De esa circunstancia surgieron nuevas oportunidades que hicieron posible mis estudios de posgrado, y me permitieron, asimismo, establecer contactos con importantes investigadores latinoamericanos, varios de los cuales formaron parte del grupo que dio origen a la Asociación Latinoamericana de Estudios del Discurso (ALED). 
OILZ: ¿Podría ampliar los comentarios sobre su inicio en el campo de la investigación en Estudios del Discurso (ED)?

AHA: Como señalaba en mi respuesta anterior, mis primeras indagaciones se enmarcaron en lo que se conoce hoy como investigación acción. En otras palabras, la búsqueda de soluciones a problemas docentes; en mi caso, a través de modelos de desarrollo curricular o de descripciones de géneros discursivos. Si tuviera que fijar un momento en el tiempo de mi dedicación a los Estudios del Discurso creo que señalaría — como fecha de inicio de mis primeras investigaciones formalizadas - los trabajos que realizara con mi colega Luz Aravena Benavente durante la década de los años ochenta del siglo pasado, antes de comenzar mis estudios de doctorado. El primero de ellos, que titulamos De la denominación de los objetos a la expresividad del discurso, produjo como resultado un libro sobre el acto de referir, que incluía una caracterización modélica de los mecanismos de producción de este acto en el corpus estudiado. El segundo proyecto - Indicios apreciativos en informes de investigación y similares - nos permitió abordar el leguaje evaluativo en un corpus de documentos en francés e inglés que publicara la Academia de Ciencias de Francia. En lo metodológico, significó poner a prueba un constructo analítico-descriptivo y aplicar un conjunto de parámetros evaluativos para dilucidar sus efectos.

OILZ: Usted cursó estudios de doctorado en la Universidad de Birmingham (Inglaterra). ¿Cómo fue esta experiencia académica? ¿De qué manera ha contribuido esta experiencia de estudio en sus trabajos investigativos?

AMH: Mi relación con la Universidad de Birmingham se inició a raíz de una invitación que hizo John McHardy Sinclair a académicos latinoamericanos para participar en sus programas de posgrado, en el marco del segundo coloquio de inglés especializado que se realizó en México. A esa invitación respondieron académicos de distintos países latinoamericanos; a modo de ejemplo: Adriana Bolívar de Venezuela y Carmen Rosa Caldas- Coulthard de Brasil.

En mi caso, el primer contacto directo con esa casa de estudios fue en el año 1983 con mi ingreso al programa de magíster; se mantuvo posteriormente con estadías de investigación y, algunos años después, con mis estudios de doctorado. Mis distintas visitas a esa institución — conocida por su investigación pionera en Análisis de Discurso y por su trabajo en lingüística de corpus - tuvo, por cierto, una influencia decisiva en mi formación académica y en mi toma de postura teóricometodológica. 
Ahora bien, los estudios de posgrado, en especial los de doctorado, no solo permiten una formación académica sistemática de nivel avanzado en una disciplina dada, sino que también proveen un espacio y un tiempo de interacción y de reflexión conjuntas entre académicos y estudiantes. Es esto último lo que más valoro de esa experiencia académica, probablemente por tratarse, en mi caso, de un doctorado a distancia. Son estas circunstancias las que me hicieron apreciar en mis períodos de residencia el contacto directo y las conversaciones con connotados y generosos especialistas, quienes compartían tanto sus conocimientos como también su tiempo.

Durante esos períodos tuve la oportunidad de trabajar directamente con Tim Johns, Michael Hoey, John McHardy Sinclair y John Malcolm Swales, entre otros, y de manera sostenida con mi director de tesis, Malcolm Coulthard. De esos períodos de residencia durante el programa de doctorado — doce semanas durante los años 1990 y 1991 y cuatro meses el año 1993 - recuerdo, en forma especial, los seminarios de posgrado de los días martes, en que distintos especialistas de otras universidades y de otros países exponían sus proyectos investigativos y se discutían sus aproximaciones; las reuniones eran a puertas cerradas con otros doctorandos, donde se presentaban y se criticaban los proyectos de tesis en curso, así como también las charlas con profesores visitantes de la talla de Michael A. K. Halliday.

A lo estrictamente académico-disciplinar quisiera agregar otras dos dimensiones que rescato como relevantes de esa experiencia: durante mi programa de doctorado tuve la oportunidad de convivir y compartir con estudiantes de posgrado provenientes de distintas culturas y realidades académicas. Algunos de ellos británicos, pero la mayoría provenientes de otros lugares, como Alemania, Australia, Brasil, China, España, Finlandia, Indonesia, Italia, Malasia, Nigeria, Sudán, Venezuela y Zimbabwe. Esta sola circunstancia es en sí formativa. Te hace valorar y comprender las diferencias, tanto culturales como académicas por una parte, y por otra, apreciar la riqueza que trae consigo el conocer distintas aproximaciones teórico-metodológicas y perspectivas investigativas de un objeto de estudio común: el discurso.

OILZ: ¿Cuáles son los principales temas y problemas que han ocupado sus intereses en Estudios del Discurso (ED)? 
AHA: Si tuviera que etiquetarlo diría que mi gran tema ha sido - y es- la ciencia como discurso, en sus variadas manifestaciones y distintos canales de circulación y consumo. En otras palabras, cómo se conceptualiza y actualiza discursivamente la (co)construcción social del conocimiento en los tres ámbitos en los cuales he indagado: (i) al interior de la comunidad científica, (ii) en la academia y (iii) en la prensa escrita. En todos ellos me ha interesado, fundamentalmente, de qué manera interactúan y se relacionan discursivamente sus diferentes actores y los recursos que despliegan para alcanzar sus propósitos comunicativos.

OILZ: Su tesis doctoral está relacionada con estos temas.

AHA: Efectivamente. En mi tesis doctoral trabajé, desde la perspectiva del Análisis del Discurso, en un corpus de informes sobre el efecto invernadero en el cual se modelaban los posibles efectos del cambio climático, caracterizando e interpretando los cambios retóricos y lingüísticos que se producían cuando estos mismos resultados eran reportados en la prensa escrita británica.

En esa misma línea trabajé, pocos años después, con Luisa Granato de Grasso en un corpus de entrevistas de divulgación científica. En ese estudio se contrastaron las entrevistas orales en audio con la correspondiente versión escrita, publicada con el propósito de ver cómo se daba el contenido informacional en una y en otra, y se reformulaba el discurso del investigador.

OILZ: ¿En qué radica la importancia de analizar la ciencia como discurso? AHA: La respuesta para mi es simple. En nuestra sociedad el conocimiento en general, y el científico en particular, es altamente valorado, por lo que en muchas instancias se utiliza como base de distintos procesos de toma de decisiones o se esgrime como herramienta de poder. Si aceptamos que todo conocimiento es discursivo, acceder al conocimiento científico implica conocer, comprender y caracterizar los diferentes discursos y géneros mediante los cuales se moviliza. Esto permite develar sus posibles efectos. Trabajo que le compete a los analistas del discurso.

OILZ: Desde su perspectiva, ¿cuáles son las grandes manifestaciones discursivas de la ciencia?

AHA: Por razones metodológicas y operacionales, divido las distintas manifestaciones discursivas de la ciencia en tres niveles que corresponden a aquellas comunicaciones de la ciencia que se crea, la que se divulga y la que se disemina, y al interior de ellas en los distintos géneros que mejor 
las representan. La primera involucra como interlocutores a los pares, los especialistas y los alumnos avanzados; la segunda, a especialistas en otras disciplinas, legos informados y estudiantes en formación; la tercera privilegia al público en general. Si bien el científico como comunicador puede dialogar con distintas audiencias, son los comunicadores sociales o los periodistas los que participan con mayor frecuencia en las dos instancias finales.

OILZ: ¿Qué efectos pueden generar estos discursos de divulgación de la ciencia en las personas?

AHA: Responder esta pregunta requeriría de varias páginas; pero daré un ejemplo para ilustrar lo anterior. Hace unos años atrás, un matutino chileno utilizó como titular la siguiente oración: "La capa de ozono se cerrará este mes". Aun cuando en el cuerpo del texto se citaban las expresiones de reputados especialistas sobre el tema, es fácil entender que la impresión que un lector no informado deducía de este titular no tenía nada que ver con la realidad.

El tratamiento del conocimiento y de sus efectos en la prensa ha sido preocupación de distintos analistas del discurso de nuestra Asociación Latinoamericana de Estudios del Discurso. A modo de ilustración, me permito señalar el libro sobre las noticias de prensa publicado hace varios años atrás por Teun A. van Dijk y los distintos proyectos que sobre el tema ha liderado Patrick Charaudeau. En nuestro continente cabe destacar los trabajos sobre la divulgación de la ciencia de María de Lourdes Berruecos Villalobos, en México y los de Guiomar Elena Ciapuscio, en Argentina, entre muchos otros.

OILZ: ¿Qué perspectiva de análisis del discurso privilegia en sus investigaciones? ¿Ha construido modelos propios de abordaje del discurso? AHA: Actualmente, en lo temático, mi interés investigativo está centrado en otra de las manifestaciones de la ciencia como discurso, en el discurso académico disciplinar oral, escrito y multimodal. Es en este discurso institucional en el que he investigado y al que he dedicado mis mayores esfuerzos en los últimos años, trabajo en el cual ha participado activamente un equipo de académicos y estudiantes del departamento de Ciencias del Lenguaje de la Pontificia Universidad Católica de Chile.

Al respecto, y como resultado de este trabajo en equipo, hemos desarrollado nuestra propia propuesta de abordaje del discurso académico, aunque llamarlo modelo creo que sería un poco pretencioso. Se trata más bien de una postura investigativa que contempla distintas

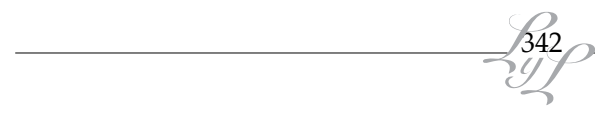


fases y un recorrido metodológico asociado que implica recabar información de distintas fuentes en forma paralela.

A modo de ejemplo, en este momento estamos estudiando el discurso de los exámenes orales finales de pregrado tomando en consideración los planteamientos institucionales, reflejados en páginas web, reglamentos, perfiles de egreso y programas de asignatura, la representación que de esta instancia evaluativa tienen sus participantes directos, académicos y estudiantes, expresada en cuestionarios, reuniones focales y entrevistas en profundidad, y en el estudio de un corpus de video-grabaciones de exámenes de distintas disciplinas, todo ello con la intención de aportar a la caracterización discursivo-interaccional de este proyecto comunicativo.

Estimamos que una aproximación de corte etnográfico en una primera etapa, como la señalada, permite en lo prospectivo una adecuada caracterización del contexto situacional, por una parte, y levantar categorías de análisis, por otra. Retrospectivamente, una vez analizadas las manifestaciones discursivas, debemos no solo interpretar sino explicar de manera más comprehensiva — con la conjunción de estas distintas voces- los resultados alcanzados.

OILZ: ¿De qué manera aporta la etnografía a los Estudios del Discurso (ED)?

AHA: Considero que la etnografía entrega antecedentes valiosos para la interpretación y la explicación de los fenómenos discursivos. Fundamentalmente, en nuestro caso, nos permite acceder a las representaciones sociales de los participantes, otorgándoles voz.

\section{Un recuento de la investigación discursiva en Chile}

OILZ: ¿De qué manera se han desarrollado los Estudios del Discurso (ED) en Chile?

AHA: Aun cuandolos Estudios delDiscurso comienzan en los años setenta del siglo $\mathrm{XX}$, los primeros trabajos aparecen en Chile prácticamente diez años después. Los Estudios del Discurso comienzan a desarrollarse en mi país en la década de los ochenta, a partir de los intentos aislados de unos pocos estudiosos provenientes de especializaciones, con propósitos específicos como la gramática del texto y del discurso, la lingüística 
aplicada, la semiótica, la sociolingüística, la pragmática, los estudios literarios, la psiquiatría, y el análisis de la comunicación y de los medios.

Durante la década del noventa, los Estudios del Discurso se fortalecieron como ámbito de investigación debido - a mi parecer- a importantes circunstancias que crearon un clima propicio: el retorno a la democracia en el país (1990), el término de la censura y de la represión sistemática y la creación de la Asociación Latinoamericana de Estudios del Discurso, ALED (1995). Durante este período se realizan, además, dos importantes reuniones científicas en nuestro medio, el primer Encuentro Nacional de Estudios Interdisciplinarios del Discurso (1997) y el tercer Coloquio de la Asociación Latinoamericana de Estudios del Discurso (1999). A ellas cabe agregar las visitas y seminarios que dictó Teun A. van Dijk, que tuvieron gran impacto en académicos y estudiantes.

Todo ello trajo consigo, durante la década del 2000, un aumento significativo del número de proyectos de investigación en discurso que se presentaron en la Comisión Nacional de Ciencia y Tecnología (CONICYT), tanto de Análisis del Discurso como en Análisis Crítico del Discurso; un incremento de las publicaciones sobre estudios del discurso en revistas nacionales; la gradual incorporación de componentes discursivos en los currículos y, por consiguiente, el creciente interés de los estudiantes por realizar seminarios de grado y tesis de posgrado en Estudios del Discurso.

Asimismo, en el año 2005 el país fue nuevamente sede de la reunión bianual de la ALED, evento al que concurrieron como expositores invitados Dominique Maingueneau, Franz van Emeren, Ingedore Koch, Carmen Rosa Caldas-Coulthard, Marianne Peronard, así como nuestros socios honorarios Teun A. van Dijk y Patrick Charaudeau, y que contó con más de seiscientos participantes. A lo anterior se añade que, también, se han llevado a cabo cinco encuentros nacionales en distintas universidades y ciudades del país; se ha producido, igualmente, un flujo constante de visitas de especialistas latinoamericanos y europeos a nuestras universidades, y se han publicado numerosos volúmenes que informan acerca de los resultados de las investigaciones realizadas.

OILZ: ¿Influyó el control y la represión de la dictadura en el desarrollo de las perspectivas críticas del Análisis del Discurso?

AHA: Ciertamente, la represión que trae consigo todo régimen dictatorial ha influido enormemente en los estudios críticos. Pero no solo ello; me 
parece que son los problemas de discriminación y desigualdad que aún persisten en nuestros países los que han despertado el interés de los investigadores.

OILZ: ¿Cuáles son los principales temas y problemas abordados por los estudiosos del discurso chilenos?

AHA: En cuanto a los temas que actualmente preocupan -o siguen preocupando - a los estudiosos del discurso chilenos se destacan, como se desprende del programa de nuestro último encuentro nacional: el discurso académico y disciplinar, el discurso de la interacción oral, el discurso gubernamental y político, el discurso de los medios, el discurso de los textos escolares, el discurso de la salud, el discurso de las minorías étnicas y el discurso de la pobreza; a estos se agregan el tratamiento de problemas tales como los derechos humanos y las diferencias de género, aquellos relacionados con ideología, identidad y cortesía lingüística, y se mantiene el interés por los géneros discursivos.

Por otra parte, si revisamos las publicaciones chilenas de estos últimos años nos encontramos, a nivel de tendencias, como características más sobresalientes con un aumento sostenido de las investigaciones en Análisis Crítico del Discurso, con un interés renovado por las interacciones orales, con nuevas perspectivas de análisis desde la multimodalidad, y con un mayor número de investigaciones que recurren a corpus electrónicos como material de estudio y análisis.

OILZ: ¿Se podría hablar de diferentes generaciones de estudiosos del discurso en Chile?

AHA: Definitivamente sí. Y ello estimo, por dos razones fundamentales. La primera tiene que ver con la formación en la disciplina que proporcionan los actuales programas de doctorado en el país - o en el extranjero-, formación sistemática de la cual carecíamos los investigadores que comenzamos a incursionar en los Estudios del Discurso y que las nuevas generaciones sí poseen. La segunda refiere al hecho de que los Estudios del Discurso tienen que ver con un momento histórico, social y político determinado, por lo cual las distintas generaciones pueden legítimamente no solo tener intereses diferentes sino que también tendrán, inevitablemente, una distinta aprehensión de esa realidad y la madurez necesaria para plantear sus propias aproximaciones.

OILZ: ¿Qué aportes han realizado los Estudios Críticos del Discurso en el análisis de fenómenos y problemáticas sociales en Chile?

AHA: Estimo que los Estudios Críticos han entregado antecedentes 
valiosos acerca de fenómenos y problemáticas sociales en nuestro medio. Así, por ejemplo, los estudios de María Teresa Oteíza Silva (2010) sobre los informes de la situación de los Derechos Humanos en Chile en el período de dictadura; los trabajos de Lésmer Antonio Montecino Soto (2010) sobre los habitantes de la calle; lo realizado por María Eugenia Merino Dickinson (2004) sobre la visión de los mapuches, quienes constituyen los indígenas chilenos, en los medios de prensa escrita, así como los trabajos pioneros de Leda Berardi Drudi (2003) desde el Análisis Crítico del Discurso, así como sus aplicaciones en el ámbito del discurso gubernamental y de los políticos — por nombrar solo algunoshan permitido develar y comprender fenómenos y también han aportado a la búsqueda de soluciones.

OILZ: ¿Qué tanta dependencia existe en Chile hacia los modelos teóricos y metodológicos de los Estudios del Discurso y los Estudios Críticos del Discurso europeos y anglosajones? ¿Cuáles son los modelos más conocidos $y$ aplicados?

AHA: Considero que el término dependencia ya no cabe cuando existe una trayectoria sostenida de más de dos décadas en una disciplina y un número importante de cultores que proponen nuevos abordajes. Yo hablaría más bien de influencias que me parecen legítimas y necesarias como sustento de base para la creación de nuevos conocimientos o como herramientas de análisis.

Por otra parte, no se puede desconocer que existen teorías, modelos y planteamientos extraordinariamente potentes que permiten explicar los fenómenos que preocupan a los analistas del discurso chilenos, algunos más recurrentes en los estudios de Análisis del Discurso y otros en el Análisis Crítico del Discurso. Entre ellos, los más influyentes y citados por los investigadores chilenos son la lingüística sistémico funcional, en su versión hallydiana y en la de la escuela de Sidney, principalmente en lo relativo a la teoría del registro y del género y la de la valoración; la teoría semio-lingüística, la teoría pragma-lingüística de la argumentación, la teoría del contexto y la de la cognición social.

Cabe señalar, asimismo — como relevantes—, los modelos que se originan desde la lingüística crítica y desde la perspectiva de la semiosis social; las perspectivas de análisis que desde la psicología discursiva aportan Derek Edwards y Jonathan Potter, entre otros; así como las de Per Linell en el marco del modelo co-constitutivo. Como aportes centrales para el Análisis Crítico del Discurso se encuentran los trabajos 
de Ruth Wodak y asociados y, por supuesto, las propuestas iluminadoras de Teun A. van Dijk. Lo anterior no significa, sin embargo, que exista un desconocimiento de los investigadores latinoamericanos y sus contribuciones, como se comprueba en citas y referencias bibliográficas, sobre todo en las tesis de posgrado.

OILZ: ¿De qué manera evalúa usted el desarrollo de los Estudios del Discurso en América Latina?

AHA: En términos generales, se podría decir que el desarrollo histórico de los Estudios del Discurso en América Latina ha sido bastante similar a lo señalado para la situación chilena; es decir, se pasa de la presencia de unos pocos esfuerzos aislados y tentativos en una primera etapa, a un posicionamiento científico como una de las disciplinas más firmes que en la actualidad se cultivan en las humanidades y las ciencias sociales. Ello se comprueba con la activa participación de analistas del discurso latinoamericanos en foros internacionales y publicaciones de renombre, así como en el prestigio y reconocimiento internacional de muchos de nuestros investigadores. Pero creo que lo más interesante de destacar son los efectos que esto ha producido al interior de nuestros países. En ese sentido, me parece que lo más notable es el reconocimiento institucional por la influencia que esto tiene sobre las nuevas generaciones. Es por ello que valoro, particularmente, el hecho de que muchas de nuestras universidades hayan incorporado a sus programas de posgrado líneas de especialización en discurso, o creado maestrías y doctorados en la especialidad.

OILZ: ¿A qué se debe que América Latina sea tal vez la región del mundo en donde más interés hay en Estudios del Discurso y sobre todo en Estudios Críticos del Discurso?

AHA: Para complementar mi respuesta anterior $-\mathrm{y}$ respondiendo directa y brevementea la pregunta-miopinión es queel interés quehan generado los Estudios del Discurso, y en especial los Estudios Críticos del Discurso en América Latina, se debe a la existencia de situaciones y problemas crónicos no resueltos en nuestros países, al creciente compromiso de académicos e investigadores por aportar en la búsqueda de soluciones mediante el análisis de representaciones, interacciones y comunicaciones que visibilicen situaciones de desigualdad y discriminación, y a una mayor conciencia de las instituciones de educación superior - al menos de algunas- de su responsabilidad social.

OILZ: ¿De qué manera evalúa el papel de la Asociación Latinoamericana de 
Estudios del Discurso (ALED) en el desarrollo de los Estudios del Discurso en América Latina?

AHA: Como presidenta de la Asociación —en los períodos 2005-2009agradezco esta pregunta que me permite plantear mis puntos de vista al respecto. Creo que la influencia de la Asociación en el desarrollo de los Estudios del Discurso ha sido fundamental. Desde su reunión fundante en Caracas —en el año 1995- la Asociación Latinoamericana de Estudios del Discurso nos ha permitido contar con un foro latinoamericano transdisciplinar, en el cual se discuten problemas teórico-metodológicos y se abordan problemáticas sociales comunes. En estas reuniones se informan y critican los distintos esfuerzos investigativos; se plantean y se conocen nuevos abordajes y posturas, y se comparte con especialistas de otras latitudes y con las nuevas generaciones. Me parece digno de destacar, asimismo, su vocación integradora. Al incentivar este punto de encuentro, la Asociación ha impulsado el establecimiento de redes de especialistas latinoamericanos. A modo de ejemplo, durante el sexto encuentro en Santiago de Chile se creó una red de estudios de la pobreza, que convoca a un número importante de investigadores de distintos países, sostiene reuniones periódicas y ha producido un número significativo de publicaciones.

Otra iniciativa de extraordinaria importancia en este proceso es la Revista de la ALED - Revista Latinoamericana de Estudios del Discursoque dirige con gran éxito Adriana Bolívar. Si bien nuestros congresos bianuales nos proporcionan un espacio que permite establecer vínculos académicos y, por cierto, crear lazos de amistad, es nuestra publicación periódica la que constituye nuestra mejor carta de presentación en la comunidad internacional.

A ello se suma el hecho de contar con una página web que ha contribuido, en lo interno, a establecer una vía rápida de contacto entre los asociados y una base de datos de los estudiosos del discurso en el continente, $y$, en lo externo, ha sentado las bases para una mayor internacionalización de la Asociación. Igualmente, la iniciativa de establecer un concurso de tesis nos permite estimular a investigadores noveles que se interesan por los estudios del discurso.

El producto de la conjunción de todos estos esfuerzos, liderados por la Asociación, no solo ha contribuido al desarrollo de los Estudios del Discurso en Latinoamérica, sino que también ha hecho de los aportes latinoamericanos en español y en portugués un referente importante de 
estudios similares realizados en otras latitudes.

Aportes a los Estudios del Discurso: Entre investigaciones y publicaciones

OILZ: En torno al discurso. Contribuciones de América Latina (2005c) ha sido uno de los libros editados más importantes publicados en Chile, puesto que logra agrupar múltiples trabajos en esta línea de estudios. Usted estuvo a cargo de su compilación. ¿Cuáles fueron las principales motivaciones para editar este texto? ¿Cómo fue la experiencia en la organización del libro? AHA: Cada editor o compilador de una obra colectiva sabe que la materialización de una instancia de creación colectiva representa solo el producto final de un largo camino, recorrido que se inicia con un proceso de toma de decisiones en el cual se imbrican intenciones, necesidades y expectativas, pero que solo se hace posible gracias a la generosidad de sus distintos participantes.

Tres fueron los aspectos fundamentales que me motivaron a emprender este proyecto. El primero de ellos tuvo que ver con la convicción de que la riqueza, la variedad y el potencial explicativo de los Estudios del Discurso constituyen una fuente indispensable de conocimiento y de evidencia empírica para todos aquellos investigadores interesados en la descripción y en el análisis de la lengua en uso. Asimismo, estos académicos contribuyen a develar los males que como sociedad nos aquejan, nos permiten escuchar a los que no tienen voz y hacen visible el compromiso del investigador con su entorno.

En segundo lugar, el Análisis de Discurso es en la actualidad un componente importante del currículo de distintas carreras del ámbito de las ciencias humanas y sociales, especialmente a nivel de posgrado. De ahí la necesidad sentida de proporcionar a nuestros estudiantes la posibilidad de contar con una compilación que diera cuenta del estado del arte de los Estudios del Discurso en Chile y en Latinoamérica, mediante el cual ellos tuvieran acceso a distintas aproximaciones teóricometodológicas, descriptivas y críticas.

Un tercer aspecto que se tomó en consideración tiene que ver con la concepción de los Estudios del Discurso como transdisciplina. El año 2002 - con ocasión de un Encuentro Nacional de Analistas del Discursoinvitamos al Presidente de la Academia Chilena de la Lengua, a algunos de sus miembros, así como al Presidente de CONICYT y al Decano de la Facultad de Artes de la Pontificia Universidad Católica de Chile, 
a participar en un panel denominado El Discurso: punto de encuentro entre las Humanidades, las Ciencias y las Artes. En esa ocasión tuvimos el privilegio de escuchar a distintos especialistas, quienes nos entregaron —desde la lingüística, la filosofía, las ciencias biológicas, las matemáticas y la música- sus respectivas y particulares visiones sobre el discurso. A partir de esta iniciativa y de este encuentro se empezó a gestar la idea de reunir estas distintas visiones en una publicación. Estas voces se complementaron y se enriquecieron con los trabajos de lingüistas y connotados analistas del discurso latinoamericanos y europeos, quienes aportaron sus personales planteamientos y reflexiones sobre este patrimonio compartido, y con la especial colaboración de Teun A. van Dijk, quien escribió el prólogo.

OILZ: ¿Qué temas y problemas abordan los estudiosos del discurso en esta compilación?

AHA: Como decía, la riqueza de los Estudios del Discurso se basa en su diversidad, tanto temática como de posturas teórico-metodológicas, así como en su capacidad para develar problemas que requieren de soluciones urgentes. Son precisamente estos aspectos los que privilegiamos para seleccionar los contenidos de los distintos capítulos que componen esta obra. El volumen que se concibió, además, como un punto de encuentro de estudiosos del discurso, provenientes de distintos campos del saber y de distintos países. El texto comprende siete capítulos y una nota final sobre el estado de la cuestión. En ellos se abordan temas como discurso y argumentación, discurso y ciencia, discurso y educación, discurso e interacción oral, el discurso de los medios y el discurso de las minorías, así como cuestiones sobre ideología, identidad, discriminación y el acceso a la palabra. En suma, en este libro quisimos, por una parte, plasmar una variedad de miradas y, por otro lado, representar en sus páginas los grandes temas que preocupan a los investigadores latinoamericanos.

OILZ: Esta compilación fue premiada por la Academia Chilena de la Lengua a la mejor obra del bienio (Premio Rodolfo Oroz, 2007). ¿Qué significó para usted esta distinción?

AHA: Valoro esta distinción porque constituye un reconocimiento de los aportes de los Estudios del Discurso a la comprensión de la comunicación humana y de la lengua en uso y, sobre todo, porque ella representa una manifestación pública de la apertura de esta institución hacia nuevas miradas y enfoques sobre los estudios del lenguaje.

OILZ: En este libro publicó el capítulo Manifestaciones evaluativas en la 
ciencia como discurso. Un estudio comparativo (Harvey, 2005a). ¿Qué planteamientos desarrolló en este escrito?

AHA: En ese artículo me refiero, en particular, a las instancias y recursos de evaluación en dos de las manifestaciones escritas que forman parte del continuum de la comunicación científica: el artículo especializado y el artículo de divulgación, con el propósito de mostrar cómo se realizan discursivamente en el texto y cuáles son algunos de sus posibles interpretativos. Para realizar esta investigación seleccionamos dos escenarios, que denominamos como el de la ciencia que se crea y aquella que se divulga, y trabajamos con un corpus de artículos representativos de ambos en dos áreas disciplinares: ciencias ambientales y ciencias médicas y biológicas. En nuestra aproximación al estudio de la evaluación adoptamos una perspectiva socio-pragmática. Así, de acuerdo a la perspectiva social, nos preocupamos en describir no sololas características lingüísticas de superficie y las retóricas de su estructuración textual, sino también de encontrar explicaciones a las razones por las cuales cierta codificación convencional de significado se considera apropiada para un contexto sociocultural institucionalizado particular. A su vez, para la descripción e interpretación de sus manifestaciones y efectos optamos por un enfoque pragmático.

Desde esta perspectiva, nos interesó entonces identificar tanto las realizaciones léxico-gramaticales, como también explorar las intencionalidades que dan origen a tales selecciones, estimar sus efectos e intentar explicar sus posibles interpretativos. Los resultados del estudio comparativo realizado nos llevaron a concluir que, conjuntamente con el cambio de situación retórica, artículo especializado versus artículo de divulgación científica, se produce en el producto textual un desplazamiento de las entidades evaluadas, así como un cambio en la organización de la información evaluativa y de los patrones de uso, con sus correspondientes selecciones discursivas, sintácticas y léxicas. Diferencias que se explican no solo por las características particulares de cada contexto e intencionalidad comunicativa, sino que - fundamentalmente - por la selección motivada de recursos persuasivos propios de una exposición analítica, en el caso de los artículos especializados y de recursos de persuasión exhortativa, en el caso de los artículos divulgativos; opciones cuyos análisis permiten mostrar cómo tales selecciones inciden directamente en la imagen de ciencia que se construye. 
OILZ: Como sabemos, uno de sus mayores intereses investigativos se orienta hacia el análisis del discurso académico. ¿En qué radica dicho interés? ¿Qué investigaciones ha desarrollado al respecto?

AHA: Las manifestaciones de la ciencia como discurso son parte integral de la formación académica disciplinar. No es de extrañar entonces que me haya interesado por el estudio de sus manifestaciones discursivas. Tampoco podemos desconocer que la equidad y la calidad de la educación superior en nuestro continente son problemas no resueltos, por lo cual me parece necesario aportar — desde la disciplina - con antecedentes fundados a este tema de discusión. Es por ello que, en estos últimos años, he focalizado mi interés investigativo en las distintas interacciones entre sus principales actores.

La primera de ellas centrada en la interacción escrita entre docentes y estudiantes, específicamente, en el producto textual 'informe', y en el análisis del material producido por estudiantes de segundo año de universidad de distintas especialidades e instituciones de la región metropolitana. La investigación nos permitió determinar las características nucleares del género en ese estadio de formación inicial e identificar las cuatro clases —o subgéneros- de informe de mayor presencia en esa etapa de desarrollo del discurso disciplinar en formación.

La segunda concierne a una instancia de estudio grupal entre estudiantes de humanidades y ciencias sociales, sesiones de trabajo orientadas hacia una meta pero con características de conversación informal. En estos intercambios verbales paritarios, los estudiantes comparten y cotejan el conocimiento disciplinar adquirido con el objeto de construir — discursivamente- significados conceptuales y metapragmáticos consensuados que conduzcan a la solución de una tarea académica común.

En la actualidad — como te mencionaba - estamos trabajando en la caracterización de exámenes finales en la formación de pregrado, también en distintas disciplinas, en un corpus de video grabaciones. De los distintos aspectos a abordar en esta investigación en curso me interesa, en lo personal, la hibridación discursiva y los roles que asumen los distintos participantes en el devenir del discurso, así como los modos semióticos y los medios que se despliegan en este verdadero juego de poder; problema que, por lo demás, ya nos planteara Michel Foucault al levantar la figura castigadora del examinador. 
OILZ: Otro de sus intereses investigativos se orienta hacia el análisis de la alfabetización disciplinar y sus manifestaciones discursivas escritas en las universidades chilenas.

AHA: Existe, hoy en día, conciencia en el nivel institucional de que los índices de fracaso y deserción en los primeros años de universidad tienen una estrecha relación con la carencia de estrategias y habilidades comunicativas requeridas para cursar estudios superiores e insertarse con éxito en las comunidades disciplinares correspondientes. Sin embargo, a mi entender, constatar el problema es solo un primer paso. Estoy convencida de que cualquier intervención didáctica que se pretenda realizar para atacar el problema implica conocer el proceso de alfabetización académica en toda su complejidad. Ello significa, primeramente, aceptar que nos enfrentamos con una práctica social en la cual convergen ideologías disciplinares, esquemas de pensamiento y variedades de lenguaje. También, es necesario entender que el proceso de aprendizaje disciplinar se plasma en las variadas interacciones discursivas en que docentes y estudiantes participan -algunas escritas y otras orales - en los distintos estadios de formación de sus respectivas comunidades de práctica. Asimismo, se requiere poder acceder y pesquisar las manifestaciones discursivas que concurren a la construcción compartida del conocimiento disciplinar con una mirada inter y transdisciplinar. Y todo ello es, sin duda, un trabajo que le compete al analista de discurso. Estas son las razones primordiales por las cuales me involucré en el campo de la alfabetización académica.

OILZ: También ha trabajado en proyectos conjuntos con investigadoras de la Universidad de La Plata - como la doctora Luisa Granato de Grasso - sobre el lenguaje de los jóvenes universitarios (Grupo ECLAR). ¿Podría plantear algunos fundamentos de estas investigaciones?

AHA: Mi participación en proyectos de investigación con académicas de la Universidad Nacional de La Plata en Argentina, también tiene su historia. Conocí a Luisa en Birmingham en el año 1984. En esa ocasión —y durante un período de casi tres meses - compartimos tanto actividades cotidianas como experiencias e intereses investigativos que condujeron, en el corto plazo, a colaboraciones institucionales. Inicialmente, en invitaciones a dictar seminarios y cursos en ambas instituciones $y$, posteriormente, en la realización de proyectos de investigación conjunta.

La primera investigación en la que colaboramos — en el contexto de un convenio macro entre ambas instituciones y específico entre facultades- 
fue el de las entrevistas de divulgación científica que comentara en una respuesta anterior. Como resultado de esta experiencia se decidió formalizar el interés manifestado por un grupo de investigadores de ambas unidades por el discurso oral y se creó, en el año 2002, el grupo ECLAR (Español de Chile y Argentina) que se planteó, entre sus objetivos, promover el estudio de las interacciones verbales en ambos países y aportar a la descripción de las respectivas variedades de español. Estas intenciones se materializaron en los años siguientes en dos nuevos proyectos que se desarrollaron a partir de lineamientos teórico-metodológicos comunes, y que abordaron la caracterización del lenguaje de los jóvenes universitarios. Con este propósito se reunió un conjunto de entrevistas semi-dirigidas, grabadas en audio, sobre temas y problemas comunes en cada país.

AHA: Por otro lado, ¿cuáles son los principales propósitos del programa EIVES (Estudios de Interacción Verbal en Estocolmo y Santiago) coordinado por usted y el doctor Lars Fant?

AHA: A partir de unos primeros contactos con Lars Fant durante el segundo y tercer coloquio de la Asociación, en Argentina y Chile respectivamente, y de una primera visita a la Universidad de Estocolmo, acordamos postular un proyecto con el propósito de estimular los estudios de la interacción verbal y promover el intercambio de estudiantes y académicos entre la entidad sueca y la Pontificia Universidad Católica de Chile. El programa EIVES, que inició oficialmente el año 2006, con el respaldo del organismo sueco STINT, ha estado funcionando durante los últimos cinco años y sus objetivos se han cumplido plenamente.

Entre sus numerosos logros cabe destacar el constante flujo de académicos y estudiantes entre ambas unidades académicas, la participación de investigadores en proyectos de investigación locales, la colaboración en docencia, la asesoría a estudiantes de posgrado en las respectivas instituciones, así como las estadías de investigación de académicos y estudiantes. A modo de ejemplo, se registra un número de veinticinco visitas de chilenos a los departamentos de español, portugués y estudios latinoamericanos, así como al departamento de inglés de la Universidad de Estocolmo.

El programa ha permitido a sus coordinadores colaborar con sus respectivos proyectos investigativos, presentar ponencias conjuntas en congresos y publicar un artículo. A ello se agrega la realización de un coloquio sobre la interacción verbal en Estocolmo en abril de 2011, 
un segundo coloquio sobre el tema que se llevó a cabo en Santiago en octubre del mismo año y la próxima publicación del libro El diálogo oral en el mundo hispanohablante. Estudios teóricos y aplicados.

Esta publicación cumple con uno de los objetivos del programa EIVES: dar a conocer y promover los estudios del diálogo en lengua española. El volumen comprende un conjunto de contribuciones teóricas, descriptivas y aplicadas, representativas de algunos de los desarrollos más recientes sobre la interacción verbal oral en español, y una revisión crítica del estado de la cuestión. Estas refieren a variados contextos del entramado discursivo de la sociedad actual —intercambios institucionales y de servicio, descripción de géneros discursivos y de conversaciones informales, entre otros- y se preocupan por ciertos mecanismos o recursos discursivos, como los operadores de debilitamiento, la deixis personal o la modalización, o dan cuenta del valor de la oralidad como manifestación cultural e identitaria en momentos socio-históricos particulares. El aporte de esta publicación se basa en la diversidad temática de las contribuciones que la componen como también en las reflexiones que las acompañan. Se agrega a ello el hecho de que sus autores provienen de tradiciones académicas, así como de países diferentes: Canadá, Chile, España, Estados Unidos, México, Noruega y Suecia.

OILZ: ¿En qué nuevos proyectos está trabajando?

AHA: Creo que uno de los proyectos más interesantes en los que he estado involucrada en estos últimos años ha sido el diseño y la implementación de un programa de doctorado en nuestra universidad. Este programa, que ha matriculado su primera cohorte este año 2011, cuenta con un componente fuerte de discurso en su currículo y ofrece - como una de sus líneas de especialización- Estudios del Discurso. En lo relativo a investigación, estoy trabajando, como comentaba en otra de mis respuestas, en los exámenes orales, investigación en curso que finaliza en el 2012.

OILZ: Finalmente, ¿qué recomendaciones daría a los estudiantes e investigadores que inician sus procesos investigativos en el campo de los Estudios del Discurso? 
AHA: Mis recomendaciones son simples y abarcan distintos planos. Como señalaba, una de las riquezas de los Estudios del Discurso se basa en su diversidad teórico-metodológica y de intereses investigativos. Ello implica para quien se inicia en la investigación conocer esa diversidad y evaluarla para poder — con conocimiento de causa - adoptar su propia postura investigativa. También significa conocer su entorno para determinar su área de interés y adoptar un compromiso con esa realidad. Junto con ello, los investigadores que se inician en este campo de especialización deben valorar los esfuerzos y aportes que desde nuestros países se han hecho a los Estudios del Discurso e incorporarlos a sus marcos de referencia. Por último, me parece importante señalar que así como es necesario tener una capacidad crítica es, igualmente, deseable en este quehacer desarrollar una permanente autocrítica.

OILZ: Dra. Harvey tiene mi agradecimiento por haber aceptado esta entrevista.

AHA: Por el contrario Oscar Iván, muchas gracias a usted por darme esta oportunidad de volver la mirada atrás y de reflexionar sobre lo que ha sido una muy, pero muy larga y gratificante carrera académica. 


\section{Referencias}

Berardi Drudi, L. (Ed.). (2003). Análisis Crítico del Discurso. Perspectivas latinoamericanas. Santiago: Frasis Editores.

Harvey Arellano, A. (2005a). Manifestaciones evaluativas en la ciencia como discurso. En torno al discurso. Contribuciones de América Latina. Chile: Ediciones Universidad Católica.

. (2005b). La evaluación en el discurso de los informes escritos por estudiantes universitarios. En Los contextos del discurso. Chile: Editorial Frasis.

(Ed.) (2005c). En torno al discurso: Contribuciones de América Latina. Chile: Ediciones Universidad Católica.

. (2006). Encuentros orales con fines de estudio: aproximaciones al tema. En Discurso, interacción e identidad. Homenaje a Lars Fant. Suecia: Universidad de Estocolmo.

. (2009). Acerca de la alfabetización académica y sus manifestaciones discursivas. En Haciendo discurso. Homenaje a Adriana Bolívar (pp. 627-645). Caracas: Comisión de Estudios de Postgrado de la Facultad de Humanidades y Educación de la Universidad Central de Venezuela.

. (2010). Los grupos de estudio: movimientos y recursos en la interacción. En Perspectivas dialógicas en estudios del lenguaje. México: Universidad Autónoma de Nuevo León.

\& Granato, Luisa G. (2000). La Entrevista de divulgación científica: Un estudio de casos. Revista Onomázein, 5, 84-94.

. (2003). La Interacción verbal de jóvenes universitarios: Estructura y secuenciación de los turnos en el español de Chile y Argentina. Revista Signos. Estudios de Lengua y Literatura, 36 (53), 77-87.

\& Muñoz, D. (2006). El género informe y sus representaciones en el discurso de los académicos. Estudios Filológicos, $41,95-114$ 
\& Fant, L. (2008). Intersubjetividad y consenso en el diálogo: análisis de un episodio de trabajo en grupo estudiantil, Revista Oralia, 11, 307-332.

\& Oyanedel, M. (2010). El grupo de estudio: conceptualización y actualización discursiva. En Alfabetización académica en el siglo XXI: Leer y escribir desde las disciplinas. Chile: Editorial Planeta Chilena.

\& Fant, L. (Eds.). (2011). El diálogo oral en el mundo hispanohablante. Estudios teóricos y aplicados. Madrid: Editorial Iberoamericana/Vervuert.

Londoño Zapata, Oscar Iván (2012). Los Estudios del Discursos: Miradas latinoamericanas I. Ibagué, Colombia: Universidad de Ibagué.

Merino Dickinson, M. E. (2004). Prejuicio étnico en el habla cotidiana de los chilenos acerca de los mapuches en la ciudad de Temuco, Chile. En Culturas e ideologías en los Andes (pp. 193-210). Quito: Editorial Abya Yala.

Montecino Soto, L. A. (Ed). (2010). Discurso, pobreza y exclusión en América Latina. Chile: Editorial Cuarto Propio.

Oteíza Silva, M. T. (2010). Patrones valorativos en el discurso oficial de Derechos Humanos en Chile: Dando valor al pasado y construyendo memorias históricas en la sociedad. Revista Discurso \& Sociedad, 4 (1), 151-183. Disponible en: http:/ / www.dissoc.org/ediciones/ v04n01/DS4(1)Oteiza.pdf 